\title{
A BIOGEOGRAFIA E A TEMÁTICA DA DIVERSIDADE CULTURAL NA EDUCAÇÃO SUPERIOR: CONTRIBUIÇÕES DA ECOLOGIA DE SABERES NA FORMAÇÃO DE PROFESSORES(AS) DE GEOGRAFIA
}

\author{
Biogeography and the cultural diversity thematic in higher education: contributions from \\ the ecology of knowledges in geography teacher eucation
}

Biogeografía y la diversidad cultural temática en la educación superior: contribuciones de la ecología del conocimiento en la formación de profesores en geografía

\author{
Ivan de Matos e Silva Junior \\ Rosiléia Oliveira de Almeida ** \\ *Doutorando PPGEFHC UFBA/UEFS - ivan.matos@ifba.edu.br. \\ ** Professora PPGEFHC UFBA/UEFS - roalmeida@ufba.br
}

Recebido em 20/10/2019. Aceito para publicação em 20/10/2019.

Versão online publicada em 10/11/2019 (http://seer.ufrgs.br/paraonde)

\section{Resumo:}

A formação de professores(as) para a diversidade cultural tem sido uma prerrogativa curricular que vem sendo lembrada com a obrigatoriedade da Lei 11.645/08, que busca, por sua vez, dar conta da história e cultura afro-brasileira e indígena. Na Geografia, essa temática encontra aderência nos estudos da geografia humana, enquanto que na geografia física, especialmente na biogeografia, o tema da diversidade cultural não tem sido adotado a contento, por conta do seu comprometimento com hermenêuticas cientificistas, em que a dimensão cultural estabelece relação de sinonímia com a ideia de um homem universal, expressa geralmente a partir das ações antropogênicas. Desse modo, o presente artigo visa apresentar contribuições teóricas da ecologia de saberes para a formação de professores(as) de Geografia, no âmbito das temáticas biogeográficas referendadas na diversidade cultural. Diante desse contexto, o presente artigo que é um dos resultados de pesquisa que vendo sendo realizada em nível de doutoramento, constitui-se como uma reflexão teórico-empírica que emergiu durante ação extensionista envolvendo estudantes, egressos e professores(as) de Geografia e Biologia. Dentre alguns resultados do estudo, reconheceu-se que a biogeografia tem forte potencial em incorporar a temática da diversidade cultural, a partir do processo de reconhecimento e visibilização de saberes e experiências dos povos originários que apresentam formas particulares de compreensão do mundo vivo em sua dimensão espaço-temporal; ajuda na superação do cientificismo no ensino de biogeografia na formação inicial e continuada de professores(as), ao mesmo tempo que assinala a importância da afirmação desses saberes como forma de superação do epistemicídio e do etnocídio.

Palavras-chave: Biogeografia. Ecologia de Saberes. Formação de Professores. Educação Intercultural. Ensino Superior.

\footnotetext{
Abstract:

Teacher education for cultural diversity has been a curricular prerogative that has been remembered with the obligation of Law 11,645 / 08, which seeks, in turn, to account for AfroBrazilian and indigenous history and culture. In Geography, this theme finds adherence in the studies of human geography, while in physical geography, especially in biogeography, the theme of cultural diversity has not been successfully adopted, due to its commitment to scientific hermeneutics, in which the cultural dimension establishes a synonymity relationship with the idea of a universal man, expressed generally from anthropogenic actions. Thus, this
} 
article aims to present theoretical contributions of the ecology of knowledges to the formation of geography teachers, within the scope of biogeographic themes endorsed in cultural diversity. Given this context, the present article, which is one of the results of research that is being conducted at the doctoral level, constitutes a theoretical and empirical reflection that emerged during an extracurricular activities involving undergraduate and graduate students, and teachers of Geography and Biology. . Among some results of the study, it was recognized that biogeography has a strong potential to incorporate the theme of cultural diversity, from the process of recognition and visibility of knowledge and experiences of native peoples who present particular ways of understanding the living world in its dimension. spacetime; It helps in overcoming scientism in the teaching of biogeography in the initial and continuing education of teachers, while pointing out the importance of affirming this knowledge as a way of overcoming epistemicide and ethnocide.

Key-words: Biogeography. Ecology of Knowledges. Teacher education. Intercultural education. University education.

\begin{abstract}
Resumen:
La formación de docentes para la diversidad cultural ha sido una prerrogativa curricular que se ha recordado con la obligación de la Ley 11.645 / 08, que busca, a su vez, dar cuenta de la historia y cultura afrobrasileña e indígena. En Geografía, este tema encuentra adherencia en los estudios de geografía humana, mientras que en geografía física, especialmente en biogeografía, el tema de la diversidad cultural no se ha adoptado con éxito, debido a su compromiso con la hermenéutica científica, en la cual la dimensión cultural establece relación de sinonimia con la idea de un hombre universal, expresada generalmente a partir de acciones antropogénicas. Así, este artículo tiene como objetivo presentar contribuciones teóricas de la ecología del conocimiento a la formación de profesores de geografía, dentro del alcance de los temas biogeográficos respaldados en la diversidad cultural. Dado este contexto, el presente artículo, que es uno de los resultados de la investigación que se realiza a nivel doctoral, constituye una reflexión teórica y empírica que surgió durante una acción de extensión en la que participaron estudiantes, graduados y profesores de Geografía y Biología. Entre algunos resultados del estudio, se reconoció que la biogeografía tiene un gran potencial para incorporar el tema de la diversidad cultural, desde el proceso de reconocimiento y visibilidad del conocimiento y las experiencias de los pueblos nativos que presentan formas particulares de entender el mundo vivo en su dimensión. espacio-tiempo Ayuda a superar el cientificismo en la enseñanza de la biogeografía en la educación inicial y continua de los docentes, al tiempo que señala la importancia de afirmar este conocimiento como una forma de superar el epistemicida y el etnocidio.
\end{abstract}

Palabras-clave: Biogeografía Ecología del conocimiento. Formación de profesores. Educación intercultural. Enseñanza superior.

\title{
1 Introdução
}

Pensar o tema da diversidade cultural na Geografia não é algo novo, uma vez que tal temática já compunha seu escopo de investigação no exercício de compreender as diversas formas de organização do espaço empreendidas por diferentes povos, algo que é, inclusive, indicado nos documentos curriculares acerca da educação geográfica. O ineditismo desse tipo de tema na Geografia encontra abrigo na possibilidade de articular a questão da interculturalidade às temáticas físicas na Geografia. Para assentar esse abrigo da diversidade cultural nas temáticas físicas, será necessária, ainda que não haja consensualidade, a adesão da Geografia à compreensão de que o tema da interculturalidade não deva localizar-

ParaOnde!?, Porto Alegre, v.12 n.2, p.207-215, 2019. http://seer.ufrgs.br/paraonde Edição Especial - III Colóquio de Pesquisadores em Geografia Física Ensino de Geografia 
se tão somente na subárea da geografia humana e, que, portanto, é preciso admitir e criar condições para que outros sujeitos, informados por outras ontologias e epistemologias, possam compartilhar seus saberes e experiências que nos informam outras geografias, e, dessa forma, várias formas de explicar o mundo, sobretudo, aquilo que se convencionou como natureza.

Com o objetivo de problematizar a necessidade de retomar as geografias vernaculares no âmbito dos estudos da natureza, partiu-se da Biogeografia, área do conhecimento com forte potencial de mobilizar saberes não apenas localizados entre as disciplinas científicas, especialmente de sua dupla filiação científica à Geografia e à Biologia (MARQUES NETO, 2018), mas destas em diálogo com outras formas de conhecimento igualmente importantes. A escolha pela biogeografia está ligada não apenas a essa condição epistemológica que aponta várias possibilidades de leituras do mundo vivo, mas, também, por integrar o atual campo de investigação do primeiro autor deste trabalho, que vem desenvolvendo a pesquisa em nível de doutoramento, intitulada "Da colonialidade à decolonialidade do saber: contribuições teórico-metodológicas das epistemologias do Sul nas aulas de Biogeografia", desenvolvido no Programa de Pós-graduação em Ensino, Filosofia e História das Ciências (UFBA/UEFS), na linha de ensino de ciências.

Como fonte de inspiração que ajudou na tessitura desse trabalho, partiu-se de resultados parciais obtidos no contexto de um conjunto de ações de natureza extensionista, que integra a pesquisa supracitada. A ação envolveu docentes da educação básica, estudantes e egressos de cursos de Geografia e Biologia, por entender que a Biogeografia atende à dupla adesão na formação inicial dessas duas áreas do conhecimento. Desse modo, o presente artigo visa apresentar contribuições teóricas da ecologia de saberes para a formação de professores(as) de Geografia, no âmbito das temáticas biogeográficas referendadas na diversidade cultural.

A presente reflexão diz respeito às contribuições teórico-empíricas da ecologia de saberes para a formação de professores(as) de Geografia, no âmbito das temáticas biogeográficas referendadas na diversidade cultural. Os resultados parciais e as discussões aqui apresentadas são fruto de reflexões que emergiram da literatura especializada em diálogo com uma pesquisa-ação (THIOLLENT, 2011) e na netnografia (KOZINETS, 2014) realizadas no âmbito do Curso de Extensão "Biogeografias do Sul: decolonizando o saber para a diversidade epistemológica do mundo", ação extensionista que integra a pesquisa de doutorado em desenvolvimento.

\section{Desenvolvimento}

Costuma-se, desde a formação inicial em cursos de formação de professores e professoras de Geografia, a defesa da garantia de que haja uma adequação do que se pratica na ciência com as demandas do contexto escolar. Essa adequação vem geralmente traduzida no que se convencionou por Geografia escolar (CAVALCANTI, 2012), a partir da compreensão de que se deva mobilizar conteúdos, metodologias e formas de avaliação que assegurem, de forma articulada, a

ParaOnde!?, Porto Alegre, v.12 n.2, p.207-215, 2019. http://seer.ufrgs.br/paraonde Edição Especial - III Colóquio de Pesquisadores em Geografia Física Ensino de Geografia 
mobilização de conceitos, temas e categorias de análise da geografia em consonância ao contexto local em que se encontram as instituições de ensino dedicadas à Educação Básica (CALLAI, 2013). A reflexão que segue aqui parte da ideia de que a ciência geográfica que é mobilizada didático-pedagogicamente no espaço escolar deva criar condições para que outros saberes sejam adotados nas práticas de ensino, compreensão esta que deve integrar desde à formação inicial docente em Geografia. No intuito de contribuir para uma formação para a diversidade torna-se oportuna a reflexão sobre como a temática da diversidade cultural é qualificada no ensino das temáticas físicas (MORAES, 2013; FIALHO,2014), especialmente nas temáticas biogeográficas

A geografia física, e especialmente a Biogeografia, sempre foi informada não apenas pelas ciências naturais, mas também pelas ciências humanas. Embora seja possível constatar adoção de abordagens que relacionem conceitos e temas das ciências naturais aos conhecimentos de povos tradicionais, o mesmo não pode ser identificado na biogeografia, que apresenta poucos trabalhos que assumem linhas de trabalho nesse viés. A geografia cultural, em especial, já havia mobilizado interesse na etnoclimatologia, etnoecologia, etnobotânica e etnozoologia, como forma de entender o conhecimento dos meios mobilizados por povos tradicionais, ao que se costuma denominar por geografia vernacular, de modo a considerar que "os saberes tradicionais frequentemente elaboram inventários admiráveis da diversidade do real" (CLAVAL, 2014, p. 234).

Em termos etnozoológicos, a assunção de que comunidades tradicionais "conhecem os hábitos alimentares dos animais de caça, os lugares onde repousam, pastam e bebem, além de seus ciclos de reprodução" (CLAVAL, 2014, p. 233) confirma a tessitura de Biogeografias, e, portanto, de formas particulares de relacionar com o mundo vivo e, por extensão, de formas particulares de responder por que uma espécie ou comunidades inteiras encontram-se em uma área e não em outra, dentre uma infinidade de perguntas que costumamos formular na Biogeografia e que encontram outras hermenêuticas. Portanto, há outras interpretações acerca das sociobiodiversidades, amparadas em saberes mobilizados pela tradição oral em seus contextos locais de vida, mesmo porque "O conhecimento acumulado por diferentes culturas que evoluíram por milhares de anos em contato direto com a natureza representa um rico patrimônio cultural com o qual a Biogeografia necessita dialogar" (FIGUEIRÓ, 2015, p. 20).

É possível reconhecer que a literatura científica em Biogeografia vem aos poucos adotando outros referenciais que ampliam possibilidades de diálogo com outros saberes, que não necessariamente encontram abrigo tão somente na Geografia e na Biologia, mas, sobretudo, nos saberes dos povos originários, na Antropologia e na Arqueologia, por exemplo. As ciências naturais ou, pelo menos, pesquisadores destas, dedicados em adotar o pluralismo cultural em suas práticas de ensino, pesquisa e extensão, já assumiram tal proposta de trabalho, especialmente a Biologia e a Ecologia, fato que vem se traduzindo na formação de grupos de pesquisa com linhas voltadas para ramos de estudos etnocientíficos, bem como no fomento de redes de pesquisadores e pesquisadoras e disponibilidade de periódicos dedicados aos estudos em etnobiologia, etnoecologia, etnobotânica, etnozoologia e etnoconservação.

ParaOnde!?, Porto Alegre, v.12 n.2, p.207-215, 2019. http://seer.ufrgs.br/paraonde Edição Especial - III Colóquio de Pesquisadores em Geografia Física Ensino de Geografia 
Nessa leitura que adota outros saberes, o que mais chama atenção é que a Biogeografia, tributária de parte da epistemologia das ciências naturais, não seguiu esse percurso dialogado com outros saberes, mesmo amparada numa área como a Geografia, que busca dar conta das manifestações e dinâmicas espaciais traduzidas pelas relações natureza e sociedade. A geografia física e, especialmente a biogeografia, poderia ter acolhido diversas epistemologias e ontologias mobilizadas por outros saberes que dialogam com o que convencionamos como natureza. Embora Claval (2014) mobilize uma leitura de resgate dos saberes de povos originários, identificando sua importância na compreensão da diversidade, opera epistemologicamente em uma gradação discursiva informada na evolução desses etnoconhecimentos para seu status de cientificidade, uma vez que concebe que "as etnotecnologias refletiam uma diversidade de saberes imperfeitamente racionalizados" (CLAVAL, 2014, p. 235) e, portanto, descritos em sua condição de precariedade e insuficiência. Esse exercício de gradação evolutiva, considerado aqui como conversão epistemológica, assenta assimetrias, instaurando a colonialidade como parâmetro de leitura dos outros, e, portanto, julgando-os a partir de referenciais e lentes consideradas fundamentais na ciência, empreendendo, assim, o desperdício de outras experiências e saberes.

Outras geografias, sobretudo aquelas que informam sobre a espacialidade do mundo vivo, precisam ser colocadas em diálogo, em que tais leituras do atrasado e do avançado se pulverizam, enriquecendo os estudos de Biogeografia, a partir de uma infinidade de hermenêuticas sobre a vida e de relações com o que convencionamos como natureza e cultura. Assumir que outras culturas mobilizam Biogeografias configura-se como uma lacuna/desafio na contemporaneidade, para os praticantes desse saber que recebe a alcunha de biogeográfico, sempre lembrado tão somente no cânone da ciência, fato que motiva a ampliá-lo para a diversidade epistemológica do mundo (SANTOS; MENESES; NUNES, 2005).

Embora não contempladas pela alcunha supracitada, várias culturas mobilizam saberes e práticas amparadas por uma infinidade de referenciais epistemológicos e ontológicos que nos apontam aspectos em que reconhecemos um tipo particular de pensamento biogeográfico. Embora uma infinidade de culturas não reconheça esse vocábulo Biogeografia, isso não impede que nos envolvamos nesse exercício de identificar, nessas culturas, elementos que informam pensamentos e práticas comunitárias que nos convidam a pensar sobre múltiplas narrativas e lentes sobre esse objeto de estudo que denominamos seres vivos. Desse modo, uma reflexão que aponta para a interculturalidade e, portanto, para o diálogo de diversas epistemologias, sugere o desenvolvimento de outras pedagogias, que venham acolher outros sujeitos, cuja produção de conhecimento tem sido silenciada. Tal proposta curricular deverá ser informada na ecologia de saberes, assumindo a condição de convivência de epistemologias e saberes práticos em diálogo com o que se pratica na ciência biogeográfica, promovendo, assim, visibilidade e dialogicidade entre as diferentes formas de conhecimento do mundo.

Propor uma abordagem pedagógica em que as temáticas biogeográficas venham acolher a diversidade epistemológica do mundo, a partir da ecologia de saberes, não é uma tarefa fácil. A dificuldade, que não deve ser vista como empecilho, encontra-se abrigada no predomínio de hermenêuticas cientificistas que assentam a ciência como via única de compreensão do mundo, tornando abissais

ParaOnde!?, Porto Alegre, v.12 n.2, p.207-215, 2019. http://seer.ufrgs.br/paraonde Edição Especial - III Colóquio de Pesquisadores em Geografia Física Ensino de Geografia 
uma infinidade de saberes e experiências, mobilizadas pela tradição oral. Os conhecimentos indígenas e afro-brasileiros informam uma teia de leituras com forte aderência ao que se convencionou como Biogeografia, geralmente informadas por outras ontologias. Tal assertiva precisa ser assumida pela ciência ou pelo menos por aqueles e aquelas que compartilham da ecologia de saberes, ou seja, de projetos políticos pedagógicos em que os diferentes conhecimentos dialoguem de forma horizontal, sem a instauração de gradações epistêmicas que venham instituir o que é superior e inferior, sob pena de empreender assimetrias e, portanto, invisibilização de outras possibilidades de leitura do mundo. O que se advoga aqui é a não superioridade epistemológica de um conhecimento que se autoproclamou como detentor da verdade. No entanto, reconhece-se que a ciência precisa dialogar com temas que ocupam certa condição de marginalidade, especialmente quanto à interculturalidade (SANTIAGO et al., 2013; CAUDAU, 2004).

A proposta de ecologia de saberes (SANTOS, 2005, 2010a, 2010b) auxilia na revisão das atuais metodologias de ensino-aprendizagem em Biogeografia na educação superior, com fortes repercussões na formação histórico-crítica do(a) docente. Esse tipo de encaminhamento sugere outra postura do professor de Biogeografia, mais horizontalizada e atenta ao aprendizado. Nesse contexto, a Biogeografia tem o desafio de admitir a diversidade de visões de mundo, reconhecendo e problematizando o potencial enriquecedor do pluralismo cultural como eixo estruturante nas práticas de ensinagem, ou seja, em que professor e estudantes ensinam e aprendem mutuamente, a partir da interlocução de saberes, abrindo, assim, a possibilidade de constituição de novos saberes no campo da interculturalidade; por entender que a superação de epistemologias cientificistas não deve convergir tão somente no plano da teoria e dos conceitos que a acompanham, mas deve incidir na forma como se empreende a ação pedagógica. Desse modo, embora a proposta das epistemologias do Sul filie-se, explicitamente, aos estudos e pesquisas no campo do multiculturalismo e suas modalidades, dentre elas o interculturalismo crítico (WASH, 2009), acolhe, sobretudo, várias características que abrigam outras experiências que assinalam formas de habitar o mundo para além das distinções entre natureza e cultura (DESCOLA, 2016).

A formação docente universitária, ao assumir a ciência como parâmetro de leitura do mundo, tem atuado no processo de invisibilização de inúmeras experiências, que são reproduzidas, por sua vez, em condições de subalternidade nos espaços acadêmicos. Tal fato tem demarcado que tais saberes só podem ser estudados fora da universidade, especialmente nos espaços em que se encontram comunidades que compartilham outras formas de habitar a Terra. Essa condição de subalternidade em que se encontram uma infinidade de saberes e experiências nos espaços acadêmicos é fruto da adoção de modelos curriculares eurocentrados, pautados, sobretudo na lógica monocultural. Assim, no âmbito das instituições de ensino superior, um projeto de decolonização do saber da formação docente é fundamentalmente uma proposta de decolonialidade curricular, em que não haja invisibilizações ou negações de outros saberes. Trata-se de um empreendimento de construção coletiva de conhecimentos, o que sugere uma nova forma de se pensar a formação de professores de Geografia.

Essa nova forma de pensar a formação docente encontrou abrigo no curso de extensão "Biogeografias do Sul: decolonizando o saber para a diversidade

ParaOnde!?, Porto Alegre, v.12 n.2, p.207-215, 2019. http://seer.ufrgs.br/paraonde Edição Especial - III Colóquio de Pesquisadores em Geografia Física Ensino de Geografia 
epistemológica do mundo". Embora se admita que a colonialidade epistêmica assentada em abordagens cientificistas ainda assuma um lugar especial e predominante na formação docente, o curso que integra a atual pesquisa de doutorado reconheceu a necessidade de desenvolver práticas pedagógicas comprometidas em visibilizar saberes e experiências com forte aderência temática com a Biogeografia. Como forma de exercitar práticas que viessem a romper uma formação cientificista, o curso adotou rodas de conversas com lideranças indígenas e afro-brasileiras, bem como especialistas de diversas formações científicas.

Além disso, como forma de decolonizar o espaço, a ação extensionista envolveu muitas atividades fora do espaço da sala de aula, como canteiros e praças da instituição, organizados de forma que todos e todas estivessem à vontade para compartilhar suas impressões sobre os textos indicados, inclusive de autoria de representantes de etnias indígenas, confrontando-os com seus saberes e experiências de vida. Tais momentos de interação não ficaram circunscritos tão somente aos encontros presenciais, mas também se estenderam aos encontros a distância, a partir do uso de plataforma online de aprendizagem, em que os cursistas tiveram a oportunidade de ampliar as discussões em fóruns de discussão, além de outras atividades realizadas nessa modalidade.

Ao invés de instituir, de forma prévia, todo o planejamento das atividades da extensão, preferiu-se traçá-las a partir das demandas e sugestões das pessoas envolvidas. A fim de criar condições favoráveis no desenvolvimento das atividades, adotou-se a exibição de vídeos e documentários que contemplavam depoimentos de quem vive a decolonialidade, além de práticas meditativas e lúdicas de produção de desenhos. O curso contou também com a vivência biogeográfica de campo em um dos poucos espaços urbanos do bioma Mata Atlântica existentes no Brasil, fortemente marcado pela presença de integrantes da tradição espiritual afrobrasileira, que mantém forte relação com as plantas, atribuindo às mesmas o sentido da sua própria existência. Esse conjunto de ações que integrou a extensão despertou nos cursistas outro olhar sobre a Biogeografia e, por extensão, outra forma de se propor trabalhos pedagógicos comprometidos em processos de visibilização de sujeitos, a partir da ecologia de saberes.

\section{Considerações finais}

A Biogeografia tem forte potencial em incorporar a temática da diversidade cultural, a partir do processo de reconhecimento e visibilização de outros saberes e experiências. Essa compreensão, que credita atenção a outros sujeitos como produtores de conhecimento, encontra na decolonialidade epistêmica a possibilidade de práticas pedagógicas comprometidas em visibilizar outras narrativas que historicamente não têm encontrado abrigo nas universidades, especialmente na subárea da geografia física.Uma proposta de formação de professores(as) informada na ecologia de saberes não representa uma ameaça à ciência, mesmo porque a ciência encontra abrigo nessa ecologia de saberes, por vezes, sempre autoproclamada como um conhecimento especial e como a melhor forma de compreender o mundo.

ParaOnde!?, Porto Alegre, v.12 n.2, p.207-215, 2019. http://seer.ufrgs.br/paraonde Edição Especial - III Colóquio de Pesquisadores em Geografia Física Ensino de Geografia 
Uma proposta de formação de professores(as) sob inspiração, portanto, na decolonialidade do saber, estará atenta a criar condições para o desenvolvimento da escuta sensível, de acolhimento da diversidade cultural manifestada nas diversas formas de ser e se relacionar com o mundo, de forma a colaborar na superação do cientificismo no ensino de Biogeografia tanto na formação inicial quanto continuada de professores(as), ao mesmo tempo que assinala a importância da afirmação desses saberes como forma de superação do epistemicídio e do etnocídio.

Os resultados e as discussões explanadas aqui integram um rol de argumentações que vem assentando uma compreensão que traz a dimensão da interculturalidade crítica no âmbito das temáticas biogeográficas ao passo que vem sendo desenvolvida uma carta de orientações em práticas decoloniais no ensino de biogeografia(a)s, no intuito de alcançar um público maior de colaboradores(as), especialmente em comunidades indígenas, quilombolas e uma infinidade de coletivos que produzem conhecimentos importantes sobre a biodiversidade. Esperase, portanto, que as reflexões aqui expostas sirvam de inspiração na revisão dos currículos de Geografia, com o fito de adotar a decolonialidade como paradigma da formação de professores e professoras para a diversidade.

\section{Referências}

ARROYO, Miguel Gonzáles. Outros sujeitos, outras pedagogias. Petrópolis, Rio de Janeiro: Vozes, 2014.

CALLAI, Helena Copetti. A formação do profissional da geografia: o professor. ljuí: Ed. Unijuí, 2013.

CANDAU, Vera Maria. Educação intercultural: entre afirmações e desafios. In: MOREIRA, Antônio Flavio; CANDAU, Vera Maria (Orgs). Currículos, disciplinas escolares e culturas. Petrópolis, Rio de Janeiro: Vozes, 2014. p. 23-41.

CAVALCANTI, Lana de Souza. Ensino de Geografia e diversidade: Construção de conhecimentos geográficos escolares e atribuição de significados pelos diversos sujeitos do processo de ensino. In: CASTELLAR, Sonia. Educação geográfica: teorias e práticas docentes. 3. ed. São Paulo: Contexto, 2012. p. 66-78.

CLAVAL, Paul. A geografia cultural. 4. ed. Tradução de Luís Fugazzola Pimenta e Margareth de Castro Afeche Pimenta. Florianópolis: Ed. da UFSC, 2014. Tradução de: La Géographie Culturelle.

DESCOLA, Philippe. Outras naturezas, outras culturas. Tradução por Cecília Ciscato. São Paulo: Editora 34, 2016. Tradução de: Diversité des natures, diversité des cultures.

ParaOnde!?, Porto Alegre, v.12 n.2, p.207-215, 2019. http://seer.ufrgs.br/paraonde Edição Especial - III Colóquio de Pesquisadores em Geografia Física Ensino de Geografia 
FIALHO, Edson Soares. As temáticas físicas e ambientais na geografia escolar. In: MAIA, Diego Corrêa (Org.). Ensino de geografia em debate. Salvador: EDUFBA, 2014. p. 91-113.

FIGUEIRÓ, Adriano. Biogeografia: dinâmicas e transformações da natureza. São Paulo: Oficina de Textos, 2015.

MORAIS, Eliana Marta Barbosa de. As temáticas físico-naturais como conteúdo de ensino da geografia escolar. In: CAVALCANTI, Lana de Souza. Temas da geografia na escola básica. Campinas/SP: Papirus, 2013. p. 1344.

SANTOS, Boaventura de Sousa; MENESES, Maria Paula; NUNES, João Arriscado. Para ampliar o cânone da ciência: a diversidade epistemológica do mundo. In: SANTOS, Boaventura de Sousa (Org.). Semear soluções: os caminhos da biodiversidade e dos conhecimentos rivais. Rio de Janeiro: Civilização Brasileira, 2005. p. 21-121.

SANTOS, Boaventura de Sousa. A ecologia de saberes. In: SANTOS, Boaventura de Sousa. A gramática do tempo: para uma nova cultura política. 3. ed. São Paulo: Cortez, 2010(a). p. 137-165.

SANTOS, Boaventura de Sousa. Para além do pensamento abissal: das linhas globais a uma ecologia de saberes. In. SANTOS, Boaventura de Sousa; MENESES, Maria Paula (Orgs). Epistemologias do Sul. São Paulo: Cortez, 2010(b).

SANTIAGO, Mylene Cristina et al. Educação intercultural: desafios e possibilidades. Petrópolis, RJ: Vozes, 2013.

WALSH, Catherine. Interculturalidade crítica e pedagogia Decolonial: in-surgir, re-existir e re-viver. In: CANDAU, V. M. Educação intercultural na América Latina: entre concepções, tensões e propostas. Rio de Janeiro: 7Letras, 2009. p. $12-42$. 